\title{
Portable Dynamic Weighing System of Yak based on BP Neural Network
}

\author{
Cai Wen, Zhengyu Xie, Yansong Deng* \\ Institute of Electrical and Information Engineering, Southwest University for Nationalities of China, \\ Chengdu, Sichuan, 610041, P.R. China \\ *Corresponding author: Email:342547288@qq.com
}

Keywords: BP Neural Network, dynamic weighing, portable type, Yak.

\begin{abstract}
In order to measure the weight of yak more conveniently and effectively, based on BP neural network, this paper provides a portable dynamic weighing System for yak. The wireless transmission mode is adopted between the acquisition module and the instrument in this system and weighing platform is added a handle and a small roller table, which overcome the shortcomings of traditional weighing station that cannot move. In order to further improve the problem of traditional weighing station's low accuracy, using the smoothing mean filter to denoise the original data and according to the output of the shear beam type weighing sensor and the speed of yak, the BP neural model is established, thus the static weight of yak being obtained. By many experiments in matlab, the results show that this system achieves the measurement accuracy of dynamic weighing system and ensures that it can be achieved technically, which has good practical value.
\end{abstract}

\section{Introduction}

Yak is a national protected animal in China. For yak breeding economic benefits, yak weight is one of the important indicators. At present, the yak weight is measured by driving the yak into the tunnel ring [1]. The entrance of the tunnel and the exit of the tunnel are provided with a door and there are multiple fences in the tunnel ring. It can separate and trap every yak. This method is easy to let the yak become restless and too cumbersome, so the accuracy is not high. To better solve the issues, this paper applies the design method of portable dynamic weighing system and the dynamic weighing algorithm based on BP Neural Network to more convenient and more accurate to measure yak weight. The measurement data is transmitted by wireless transmission mode, which reduces the limitation of the mobile range. In addition, in order to make the electronic weighing more portable, this system designed the wheel and handle on the weighing platform. Before using the neural network algorithm, we use the smoothing mean filter to filter out peak pulse and periodic interference. Then using BP network algorithm to good self-learning ability to train a large number of measured data. It can always find a suitable network model to calculate the exact weight of the measured yak.

\section{Portable dynamic weighing System.}

The portable dynamic weighing system consists of shear beam type weighing sensor, A/D conversion module, STM32, wireless module and computer. The links between the modules are as follows:

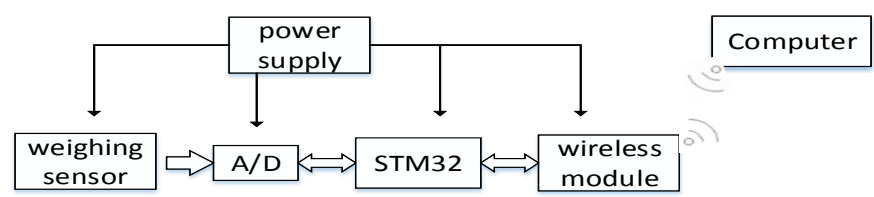

Fig. 1 Structure of dynamic weighing system

As shown in Figure 1, weighing sensor converts the collected pressure signal into the electrical signal output. Through the conversion of the A/D module, analog electrical signal is converted into digital signal. The digital signal is processed by the algorithm in STM32 to obtain the final data results, and then send the results to the wireless module, the wireless module to the computer for display and 
save. The computer can also send commands to the lower machine through the wireless module to control.

As shown in Figure 2, in the portable dynamic weighing system, the distribution structure of the weighing sensor is introduced in detail. The portable dynamic weighing system with 2 weighing platforms and 4 shear beam type weighing sensors. There are two sensors in each weighing platform. Two small rollers are placed under each weighing platform. Before weighing, first finding a flat terrain, the two will be weighing platforms separated from the appropriate distance, and then place the carrying plate on the two weighing platforms. When the yak walks through the weighing platform along the direction of the picture, the corresponding weight data can be obtained.

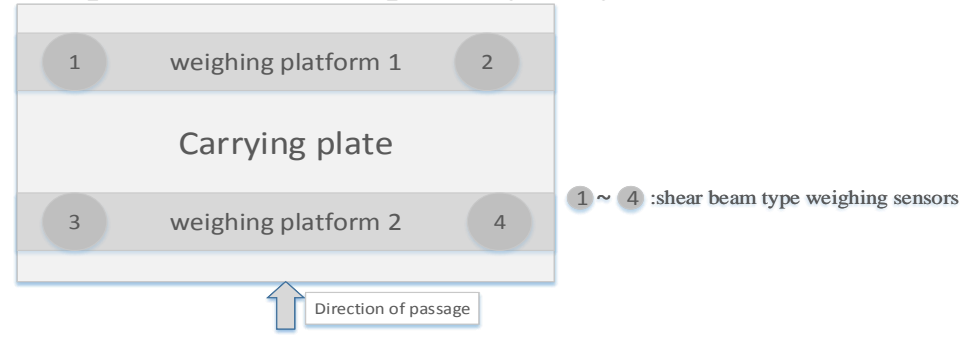

Fig. 2 The distribution structure of the weighing sensor

\section{Smoothing mean filter}

According to the characteristics of the dynamic weighing signal, it is necessary to prevent the periodic interference of noise and filter out the peak signal. Because yak speed is not very high in general, it can be processed by smoothing mean filter. The first stage of the algorithm, first remove the maximum and minimum values of this group of data, and then do the arithmetic mean operation. The second array is also used to remove the most value and get the arithmetic average value. The input of the second array is the result of the first stage, and the output is the final result.

\section{BP neural network}

\subsection{BP network learning rules}

After the original axle load signal is processed by smooth mean filtering, and BP neural network is established to obtain more accurate static vehicle weight. The generation of BP network is attributed to the BP algorithm. BP algorithm belongs to the $\delta$ algorithm, and it is a supervised learning algorithm. BP network works in general, that is, by constantly adjusting the layers and layers between the various weights, making the error function minimum. In the forward transmission of information, the output of the ith neuron in the hidden layer is shown in Equation 1:

$$
\mathrm{a}_{1 i}=f_{1}\left(\sum_{j=1}^{r} w_{1 i j} \mathrm{p}_{j}+b_{1 i}\right), i=1,2, \ldots ., \mathrm{r}
$$

The output of the k-th neurons in the output layer is shown in Equation 2:

$$
\mathrm{a}_{2 i}=f_{2}\left(\sum_{j=1}^{s 1} w_{2 k i} \mathrm{a}_{2 i}+b_{2 k}\right), k=1,2, \ldots \ldots, \mathrm{s}_{1} \text {. }
$$

Define the error function as Equation 3:

$$
\mathrm{E}(\mathrm{W}, \mathrm{B})=\frac{1}{2} \sum_{k=1}^{s 2}\left(t_{k}+\mathrm{a}_{2 k}\right)
$$

In the case of using the gradient descent method to change the weight of the change and error, the weight of the output layer changes to the weight from the ith input to the kth output as follows:

Where:

$$
\begin{aligned}
& \Delta \omega_{2 k i}=-\eta \frac{\partial E}{\partial w_{2 k i}}=-\eta \frac{\partial E}{\partial a_{2 k}} \times \frac{\partial a_{2 k}}{\partial w_{2 k i}} \\
= & \eta\left(t_{k}-a_{2 k}\right) f_{2}^{\prime} \times a_{1 i}=\eta \delta_{k i} a_{1 i}
\end{aligned}
$$

Similarly, getting the following:

$$
\delta_{k i}=\left(t_{k}-a_{2 k}\right) f_{2}^{\prime}, e_{k}=t_{k}-a_{2 k}
$$




$$
\begin{aligned}
\Delta b_{2 k i}= & -\eta \frac{\partial E}{\partial w_{2 k i}}=-\eta \frac{\partial E}{\partial a_{2 k}} \times \frac{\partial a_{2 k}}{\partial w_{2 k i}} \\
& =\eta\left(t_{k}-a_{2 k}\right) f_{2}^{\prime}=\eta \delta_{k i}
\end{aligned}
$$

Implicit layer weight change: The weights from the jth input to the ith output are shown below:

Where:

$$
\begin{aligned}
& \Delta w_{1 i j}=-\eta \frac{\partial E}{\partial w_{1 i j}}=-\eta \frac{\partial E}{\partial a_{2 k}} \times \frac{\partial a_{2 k}}{\partial w_{1 i}} \times \frac{\partial a_{1 i}}{\partial w_{1 i j}} \\
& =\eta \sum_{k=1}^{s 2}\left(t_{k}-a_{2 k}\right) f_{2}^{\prime} \times f_{1}^{\prime} \times p_{j}=\eta \delta_{i j} p_{j}
\end{aligned}
$$

Similarly, getting the following:

$$
\delta_{i j}=e_{i} f_{1}^{\prime}, e_{i}=\sum_{k=1}^{s 2} \delta_{k i} \delta w_{2 k i}, \delta_{k i}=e_{k} f_{2}^{\prime}, e_{k}=t_{k}-a_{2 k}
$$

$$
\Delta b_{1 i}=\eta_{i j}
$$

\subsection{The Determination of BP Neural Network Structure}

This paper selects the three-tier BP network from the input layer, the hidden layer and the output layer. For a BP network, the selection of its input will directly determine the ability of the network to finalize data, so the first to determine the input neurons. From experiment, it can be found the weight signal of yak is related to the speed. The higher the speed, the steep rise of the gravitational signal, and the farther the value of the ascending segment deviates from the true value of yak. In the case of a static weight of yak, passing weighing sensor at different speeds, the resulting dynamic weight values are different. Therefore, the dynamic weight and speed of yak in weighing platform 1, the dynamic weight and speed of yak in platform 2, as the input of the BP network, the number of input nodes is 4.

Yak speed can be measured using weighing sensor. When the measured yak through the weighing sensor, the pressure through the AD conversion sampling points to sum. Since the sampling rate $f_{c}$ can be set by the microcontroller STM32, the time of each beef hoof passing through the weighing sensor can be derived from the total number of sampling points. The effective width $\mathrm{d}$ of the weighing sensor is determined. This can be calculated the speed at which one of the hoofs passes through the weighing sensor. Finally, the average speed of each hoof, get yak speed, it's $V_{i}=d_{c} / n_{i}$. Where $V_{i}$ is the speed of the ith cow hoof. $d$ is the effective length of weighing sensor. $n_{i}$ is the number of data loaded for the ith beef hoof. $\mathrm{f}_{\mathrm{c}}$ is the data sampling rate.

The purpose of dynamic weighing is to measure its static weight in the yak movement, so the expected output of the network is the static weight of the yak w, the number of output nodes is 1 .

Finally, the number of hidden layer nodes is determined. According to the equation: $\mathrm{n}=\sqrt{n_{i}+n_{0}}+\mathrm{a}, \mathrm{n}=2 \mathrm{n}_{\mathrm{i}}+1$. Where, $\mathrm{n}_{\mathrm{i}} \mathrm{n}_{\mathrm{o}}$ are the number of input nodes and the number of output nodes. $\mathrm{a}$ is a constant between. 1 and 10. The number of hidden nodes can be given an initial value according to Eq. (1) or (2), and then stepwise growth or gradual pruning. The number of hidden layer nodes is $9 \sim 13$, and the number of hidden layer nodes in the network model is 12 .

Through the determination of the number of network layers and the number of input and output nodes and the selection of hidden layer nodes, the network structure is $4 \times 12 \times 1$.

\section{Experiments and analysis.}

In order to verify the theory's effectiveness and correctness, the author does the simulation experience in MATLAB. We mainly choose the bulls for testing, including bullock and ordinary bulls. Respectively, to test and calculate their weight and speed after their through weighing platform one and weighing platform two. A total of 100 samples of sample data were collected, of which 92 were used as training samples and 8 groups as test samples. Before the validation, the training samples are normalized using equation $\mathrm{x}_{\mathrm{i}}=\frac{x_{i}-x_{\min }}{x_{\max }-x_{\min }}$, and the input values are quantized into the range $[0,1]$. 


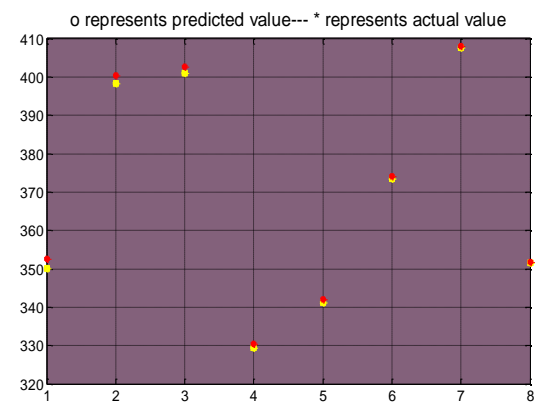

(a)

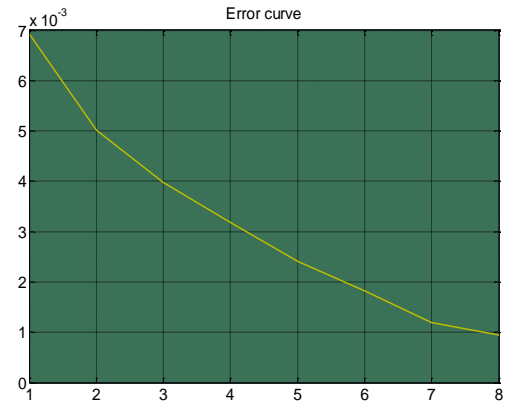

(b)

Fig.3 The comparison of the actual weight and the predicted weight of the male yak

In Fig.3 (a), we take the weight of yak in weighing platform 1 and 2, the speed of yak in weighing platform 1 and weighing platform 2 as a BP network input, the yak static weight as the BP network output, Finally, eight sets of data were obtained from the network test after training. From the figure we can see the predicted value and the actual value is basically the same, little difference, achieved a better expected effect. It can be seen from Fig. 3(b) that all errors are kept within 3\% after BP network processing, and the error of the dynamic weight of yak tends to increase with the increase of yak velocity. Obviously, the dynamic weighing model based on BP neural network has a good effect.

\section{Conclusion.}

In this paper, for the yak weight measurement of the cumbersome and inaccurate phenomenon, providing with a portable dual weighing platform weighing system to improve. The use of lightweight dual platform and data wireless transmission mode to make the operation more convenient. The static weight of dynamic yak can be obtained based on the good self - learning ability of BP neural network, which has a good application prospect.

\section{Acknowledgements}

This work was partially supported by National Nature Science Foundation (61673016), SWUN Construction Projects for Graduate Degree Programs (2017XWD-S0805), Advance Research Program of Electronic Science and Technology National Program (2017YYGZS16) and Sichuan Youth Science and technology innovation research team (2017TD0028), also was financially supported by students innovation training project of SWUN (No. 201610656038).

\section{References}

[1]Pinggui Yang, Weisheng Wu, Tianwu An. Yak roadway: CN, CN 203457612 U[P]. 2014.

[2]Xiaodong Zhang, Lihong Li,Y anqin Song. Vehicle Dynamic Weighing Algorithm Based on Double Scale Platform Truck Scale [J]. Sensors and Microsystems, 2015,(06):133-136.

[3]Junhui Zhang, Linian Wang. Research on Dynamic Weighing Algorithm [J]. Weighing, 2014,(07):39-43+55.

[4]Weitao Wang. Research and Design of Dynamic Weighing System for Wireless Portable Vehicles [D]. XiAn Industrial University, 2013.

[5]Xingfeng Cao. Research on Application of BP Neural Network in Meteorological Fore Science and Technology Bulletin cast [J]., 2012,(08):55-57.

[6]Qingqin Wang. Research on Vehicle Dynamic Weighing System Based on Intelligent Algorithm [D]. Zhejiang University, 2006.

[7]Hongli He, Yuan Zhang, Yunpeng Lv. Application of Neural Network in Dynamic Weighing Data Processing of Automobile [J]. Automated instrumentation, 2007, (05):17-19. 